Pacific Journal of Mathematic 


\title{
ALGEBRAIC GEOGRAPHY: VARIETIES OF STRUCTURE CONSTANTS
}

\author{
F. J. Flanigan
}

Let $\mathscr{C}=\mathscr{C}(n, \Omega)$ denote the algebraic set of structure constants for $n$-dimensional associative algebras, a subset of $\Omega^{n^{3}}$. Here $\Omega$ is a universal domain over a prime field $\boldsymbol{F}$ and a point $c=\left(c_{h i j}\right)$ with $h, i, j=1, \cdots, n$ is in $\mathscr{C}$ if and only if the multiplication $\left(x_{h}, x_{i}\right) \rightarrow x_{h} x_{i}=\sum_{j} c_{h i j} x_{j}$ is associative. The set $\mathscr{C}$ is readily seen to be $F$-closed in the Zariski topology on $\Omega^{n 3}$ and is in fact a finite union of irreducible closed cones (the components of $\mathscr{C}$ ) with the origin as vertex. The natural "change of basis" action of the group $G=G L(n, \Omega)$ on $\mathscr{C}$ yields a one-one correspondence between orbits $G \cdot c$ on $\mathscr{C}$ and $n$-dimensional $\Omega$-algebras. One studies the globality of these algebras (and of algebras defined over subfields of $\Omega$ ) by examining the geography of $\mathscr{C}$.

Thus if $S$ is a semi-simple $\Omega$-algebra (more generally, if the Hochschild group $H^{2}(S, S)=(0)$ ) then its corresponding orbit (denoted $G \cdot S$ ) is open and therefore dense in its component $\mathscr{C}$ of $\mathscr{C}$. Thus $S$ determines all algebras which live on $\mathscr{C}_{0}$. One checks that $\operatorname{dim} \mathscr{C}_{0}=n^{2}-n+s$, where $S=$ $S_{1} \oplus \cdots \oplus S_{s}$ for simple $S_{\alpha}$. Moreover, in the language of Gerstenhaber and Nijenhuis-Richardson, one may hope to deform the algebras on $\mathscr{C}_{0}$ into $S$. In commencing a study of the parameter space $\mathscr{C}$, therefore it seems a natural first question to ask whether every irreducible component of $\mathscr{C}$ is dominated by such an open orbit or, in the sense of deformation theory, "Does every algebra deform into a rigid algebra?" We show here that the answer is no.

In $\S 1$ below, we develop some relations between deformation of algebras and specialization of points on $\mathscr{C}$. In $\S 2$ our question is partly settled by a demonstration that every component of $\mathscr{C}$ must carry an open subset of nonsingular points which is either the orbit of a single rigid algebra or an infinite union of orbits of $\Omega$-algebras which differ only in their radicals. Then in $\S 3$ we answer the question in the negative, showing that the second alternative of $\S 2$ does in fact occur by exhibiting a full component of $\mathscr{C}$ which consists entirely of the orbits of three-dimensional nilpotent algebras.

The author recalls with pleasure several discussions with Professors Murray Gerstenhaber, Albert Nijenhuis, Alan Landman and Maxwell Rosenlicht during the course of this work.

1. Deformation and specialization. Throughout this paper, $k$ 
will denote a subfield of the universal domain $\Omega$ in the sense of Weil [6]. Let $c=\left(c_{h i j}\right)$ be a $k$-rational point of $\mathscr{C}$, thereby determining an $n$-dimensional $k$-algebra $A$ with multiplication $\left(x_{h}, x_{i}\right) \rightarrow x_{h} x_{i}=$ $\sum_{j} c_{h i j} x_{j}$ which we denote $\operatorname{alg}(c, k)$. We say moreover that $c$ is a structure for $A$. A deformation of $A$ in the sense of Gerstenhaber [2] is then given by an $n^{3}$-tuple $\kappa=\left(\kappa_{h i j}\right)$ of power series $\kappa_{h i j}=$ $\kappa_{h i j}(t)$ in a single indeterminate $t$ which satisfies (i) the multiplication $\left(x_{h}, x_{i}\right) \rightarrow x_{h} * x_{i}=\sum_{j} \kappa_{h i j} x_{j}$ is associative and (ii) $\kappa_{h i j}(0)=c_{h i j}$. Such a new multiplication determines a $K-(=k((t))-)$ algebra $A_{t}=$ $\operatorname{alg}(\kappa, K)$; we say that $A$ has been deformed into $A_{t}$. Any $k$-imbedding $k(\kappa) \rightarrow \Omega$ yields an $n^{3}$-tuple $\bar{c}$ which is a point of $\mathscr{C}$ (and thereby determines an $n$-dimensional $k(\bar{c})$-algebra.) Further, the substitution $t \rightarrow 0$ induces a $k$-specialization $\bar{c} \rightarrow c$ rational in the sense that $c$ is a rational point for $k$. Thus deformation implies specialization.

Conversely, it is asked [2, p. 85] if every such specialization in $\mathscr{C}$ is induced by a deformation. That is, let $c, \bar{c} \in \mathscr{C}$ with $c$ rational over $k$ and let $\bar{c} \rightarrow c$ be a $k$-specialization. Do there exist $n^{3}$ power series $\kappa_{h i j} \in k[[t]]$ such that (i) $\kappa_{h i j}(0)=c_{h i j}$ and (ii) the map $\kappa_{h i j} \rightarrow \bar{c}_{h i j}$ defines a $k$-isomorphism $k[k] \rightarrow k[\bar{c}]$ ? If such is the case, then we say $\kappa$ and $\bar{c}$ are k-equivalent over $c$. Moreover, we would rather not rule out the cases where $k$ is an arbitrary subfield of $\Omega$, or $c$ is a singular point on loc $(\bar{c}, k)$, the $k$-locus $(=k$-closure) of $\bar{c}$. The lemmas below (which are independent of $\mathscr{C}$ ) give us sufficient information to proceed in $\S 2$ and $\S 3$. More immediately, however, we are enabled to give an answer (complete but for a field of definition question) to the problem "Does a rigid algebra remain so after extension of scalars?"

Lemma 1. Let $\bar{x} \rightarrow x$ be a rational k-specialization in $\Omega^{m}$. Then there exist $m$ power series $\kappa_{j} \in k[[t]]$ such that $\kappa=\left(\kappa_{j}\right)$ and $\bar{x}$ are $k$ equivalent over $x$ provided one of the following holds:

(A) $x$ is a simple point on the k-variety loc $(\bar{x}, k), k$ arbitrary,

(B) loc $(\bar{x}, k)$ is a curve, $k$ arbitrary,

(C) $k=C$, the complex numbers.

Proof. (A) Write $X=\operatorname{loc}(\bar{x}, k)$. Then $k[\bar{x}]$ is a $k$-subalgebra of $\mathfrak{v}=\mathfrak{v}(x, X, k)$, the local ring of $k$-rational functions on $X$ defined at $x$. Since $x$ is simple on $X$, Cohen's Theorem [7, p. 307] assures us that the completion $\hat{\mathfrak{D}}$ of $\mathfrak{D}$ is isomorphic to $k\left[\left[t_{1}, \cdots, t_{d}\right]\right]$, where $d=\operatorname{dim} X$. Without loss then each $\bar{x}_{j}$ is a power series $\xi_{j}\left(t_{1}, \cdots, t_{d}\right)$ with constant term $x_{j}$. Now choose power series $\theta_{1}, \cdots, \theta_{d}$ in a single variable $t$ which are algebraically independent over $k$ and satisfy $\theta_{h}(0)=0$. Then the required $\kappa_{j}$ are given as $\kappa_{j}(t)=\xi_{j}\left(\theta_{1}(t), \cdots, \theta_{d}(t)\right)$. 
(B) This was noted in [2, p. 85]. Let $X^{*} \rightarrow X$ be the usual normalization. Then as above we have $k[\bar{x}]$ a subalgebra of $\hat{\mathfrak{v}}=$ $\hat{\mathfrak{b}}\left(x^{*}, X^{*}, k\right)$ where $x^{*}$ is any point over $x$. Since $X^{*}$ is simple in codimension one, $\hat{\mathfrak{D}}$ is isomorphic to $k[[t]]$ and the lemma follows.

(C) By modifying the Theorem of the Primitive Element as given in $\left[4\right.$, p. 126], we have without loss $k\left[\bar{x} \mid=k\left[\bar{x}_{1}, \cdots, \bar{x}_{d}\right][\eta]\right.$ with $d=$ $\operatorname{dim}(\bar{x}, k)$. Let $\kappa_{1}, \cdots, \kappa_{d}$ be algebraically independent complex power series in a variable $\tau$ satisfying $\kappa_{j}(0)=x_{j}$. Since $k=C$, the Puiseaux Theorem [5, p. 98] yields a $k$-algebra isomorphism $k\left[\bar{x}_{1}, \cdots, \bar{x}_{d}\right][\eta] \rightarrow$ $k\left[\kappa_{1}, \cdots, \kappa_{d}\right][\theta]$ where $\theta$ is a power series in $\tau^{1 / n}$ for some $n$. Replace $\tau$ by $t^{n}$ and the result follows.

In conjunction with case (B) above, the following intuitive result will be useful.

Lemma 2. Let $X$ be an affine k-variety in $\Omega^{m}$ and $Z$ a proper $k$-closed subset containing a k-rational point $x$. Then there exists a $k$-closed curve $\Gamma$ in $X$ through $x$ such that $\Gamma \cap Z$ is finite.

One may prove this by restricting to a suitable $k$-hypersurface in $X$ and applying induction.

An immediate application: let $\mathscr{C}_{0}$ be a component of $\mathscr{C}$. Let $c \in \mathscr{C}_{0}$ be $k$-rational and $A=\operatorname{alg}(c, k)$. Then it is standard that the orbit $G \cdot A(=G \cdot c)$ is locally closed (open in its closure) in the Zariski topology on $\mathscr{C}_{0}$. Let $k_{0}$ be the minimal field of definition for $\mathscr{C}_{0}$. (It is thought that $k_{0}=\boldsymbol{F}$, the prime field, but this has not been established.).

Proposition 3. Let the subfield $k$ contain $k_{0}$. Then the k-algebra $A$ is rigid over $k$ in the sense of Gerstenhaber if and only if its orbit $G \cdot A$ is open in the Zariski topology on $\mathscr{C}_{0}$.

Remark. Nijenhuis-Richardson [3] use “open orbit” to define rigidity; their concern is almost entirely with $\Omega$-algebras. Proposition 3 implies:

COROLlary 4. Let $k, k_{0}$ be as above. Then $A$ is rigid over $k$ if and only if the algebra $A_{k^{\prime}}$ is rigid over $k^{\prime}$, where $k^{\prime}$ is any extension of $k$ in $\Omega$.

Proof of Proposition 3. First let $A$ be rigid over $k$ and let $Z$ denote the closure of $G \cdot A$ in $\mathscr{C}_{0}$. If $Z=\mathscr{C}_{0}$, we are done. Assume not; then Lemma 2 allows us to run a curve $\Gamma$ through $c$ whose $k$ generic point $\bar{c}$ is not in $Z$. The $k$-specialization $\bar{c} \rightarrow c$ yields, by 
Lemma 1, a nontrivial deformation of $A$ into the algebra determined by $\bar{c}$, contradicting rigidity. Thus $Z=\mathscr{C}_{0}$.

Conversely, if $G \cdot A$ is open, then it is $k$-open since $c$ is $k$-rational. But any deformation of $A$ over $k$ will induce a $k$-specialization $\bar{c} \rightarrow c$. Thus $\bar{c} \in G \cdot A$ and the deformation is trivial, completing the proof of the theorem.

2. Open sets of algebras. Now we offer a preliminary answer to the question "Does every component of $\mathscr{C}$ carry an open orbit?" In what follows we will express an algebra $A$ as a semi-direct sum $N \oplus S$ of its radical $N$ and a separable Wedderburn factor $S$.

Theorem 5. Let $\mathscr{C}_{0}$ be a component of $\mathscr{C}$ with field of definition $k_{0}$. Then $\mathscr{C}_{0}$ contains a $k_{0}$-open subset $\mathscr{K}_{0}$ consisting of nonsingular points which is either the orbit of a rigid algebra or an infinite union of orbits of $\Omega$-algebras $A, A^{\prime}, \cdots$ with the following properties: if $A=N \oplus S$ and $A^{\prime}=N^{\prime} \oplus S^{\prime}$ then

(i) $S$ and $S^{\prime}$ are isomorphic as $\Omega$-algebras,

(ii) $\operatorname{dim} N=\operatorname{dim} N^{\prime}$ and this is minimal for algebras on $\mathscr{C}_{0}$,

(iii) nilindex $N=$ nilindex $N^{\prime}$ and this is maximal for algebras on $\mathscr{C}_{0}$,

(iv) the two-sided regular actions of $S$ and $S^{\prime}$ on $N$ and $N^{\prime}$ respectively are equivalent,

(v) $\operatorname{dim} H^{q}(A, A)=\operatorname{dim} H^{q}\left(A^{\prime}, A^{\prime}\right)$ (Hochschild cohomology) for a specified set of indices $q$ and, moreover, these dimensions are minimal on $\mathscr{C}_{0}$,

(vi) the orbits $G \cdot A, G \cdot A^{\prime}, \cdots$ all have the same dimension.

REMARK. The algebras $A, A^{\prime}, \cdots$ must have nonisomorphic radicals $N, N^{\prime}, \cdots$. In $\S 3$ we shall exhibit a component $\mathscr{C}_{0}$ which does in fact carry such a family of algebras.

Proof. To obtain (ii), (iii) and (v) we will employ the uppersemi-continuity principle illustrated in [2, p. 90]. Then (i) and (iv) will be derived using the results of the last section.

We will use upper semi-continuity in the following form: let $f: X \rightarrow Y$ be a dominant morphism of varieties, and let $Y_{q}$ be the set of $y$ in $f(X)$ such that $\operatorname{dim} f^{-1}(y) \leqq q$. Then $Y_{q}$ is open in $Y$.

We restrict consideration to the open subset of nonsingular points on $\mathscr{C}_{0}$. The upper semi-continuity argument of [2] showed that the subset $Y$ of structures in $\mathscr{C}_{0}$ with minimal dimensional radical is open, giving (ii). Now we deal similarly with the nilindex of the radical. For a natural number $\nu$, form the product $Y \times\left(\Omega^{n}\right)^{\nu}$. Let $X^{\prime}$ denote the subset of points $\left(c, z_{1}, \cdots, z_{\nu}\right)$ where each $z_{i}$ is an element of $N(c)$, the radical of $\operatorname{alg}(c, \Omega)$, here identified with $\Omega^{n}$. There is a poly- 
nomial map $\mu: X^{\prime} \rightarrow \Omega^{n}$ given by $\left(c, z_{1}, \cdots, z_{\nu}\right) \rightarrow z_{1} \cdots z_{\nu}$ with the multiplication determined by $c$. Denote by $x$ the closed subset $\mu^{-1}(0)$ of $X^{\prime}$. Let $\pi: X \rightarrow Y$ be the projection $\left(c, z_{1}, \cdots, z_{\nu}\right) \rightarrow c$. We note that if nilindex $N(c) \leqq \nu$, then $\operatorname{dim} \pi^{-1}(c)=\nu \cdot \operatorname{dim} N(c)$, while if nilindex $N(c)>\nu$, then $\operatorname{dim} \pi^{-1}(c)<\nu \cdot \operatorname{dim} N(c)$. By upper semi-continuity the set $Y_{1}$ of structures $c$ which determine radicals $N(c)$ of nilindex larger than $\nu$ is open in $Y$, giving (iii).

Now we obtain an open subset $Y_{2}$ of $Y$ by considering Hochschild cohomology. For $q \geqq 0$, let $C^{q}=C^{q}\left(\Omega^{n}, \Omega^{n}\right)$ denote the $\Omega$-space of $q$ cochains; this is isomorphic with $\left(\Omega^{n}\right)^{q+1}$. Proceeding as above we form $Y_{1} \times C^{q}$ and define a polynomial map $\delta: Y_{1} \times C^{q} \rightarrow C^{q+1}$ by $(c, \varphi) \rightarrow \delta_{c} \varphi$ where $\delta_{c}$ denotes the $q^{t h}$ coboundary operator for alg $(c, \Omega)$. Let $X$ be the subset $\delta^{-1}(0)$ of $Y_{1} \times C^{q}$ and let $\pi: X \rightarrow Y_{1}$ be the projection $(c, \phi) \rightarrow c$. Then the fiber $\pi^{-1}(c)$ is isomorphic with $Z^{q}(c)$, the $\Omega$-space of $q$-cocycles for $\operatorname{alg}(c, \Omega)$. Since $\operatorname{dim} Z^{q-1}(c)$ determines $\operatorname{dim} B^{q}(c)$, it is clear that there is an open subset $Y_{2}$ of $Y_{1}$ consisting of those structures $c$ for which $\operatorname{dim} H^{q}(c)$ is minimum on $Y_{1}$ : in fact, this is true for any specified finite set of indices $q$. Thus we have established (v). Our work thus far underscores the fact that a rigid algebra (open orbit) on $\mathscr{C}_{0}$ would have the "smallest" radical and second cohomology among nearby algebras.

Now we return to the methods of deformation theory to obtain (i) and (iv). We show in fact that all algebras which live on $Y_{1}$ above have isomorphic Wedderburn factor $S$ and isomorphic actions of $S$ on the radical. Let $c, c^{\prime}$ be structures in $Y_{1}, k$ a common field of definition for $c, c^{\prime}$ and $\mathscr{C}_{0}$, and $\bar{c}$ a $k$-generic point for $\mathscr{C}_{0}$. Then $\bar{c}$ is in the $k$-open set $Y_{1}$ and by $\S 1$ we may deform both $\operatorname{alg}(c, k)$ and $\operatorname{alg}\left(c^{\prime}, k\right)$ into the algebra determined by $\bar{c}$. Now one may consider the Wedderburn factor of an algebra to be rigid under deformations of the algebra, and similarly for the actions of the factor on the radical (cf. Theorem 1 of [1]). It follows after extension of scalars to $\Omega$ that the Wedderburn factors determined by $c$ and $c^{\prime}$ are each subalgebras of alg $(\bar{c}, \Omega)$. But on $Y_{1}$ all Wedderburn factors have the same dimension and hence all are isomorphic, giving (i). Statement (iv) follows from the rigidity of the actions of the Wedderburn factors.

It is standard that there is an open subset $Y_{3}$ of the transformation space $\mathscr{C}_{0}$ on which all orbits have the same maximal dimension. Putting $\mathscr{K}_{0}=Y_{2} \cap Y_{3}$ gives the theorem.

3. A component without open orbit. Now we demonstrate that the second alternative of Theorem 5 does occur in nature. Let $\mathscr{6}=\mathscr{C}(3, \Omega)$ be the algebraic set of structures for three-dimensional algebras over an arbitrary universal domain $\Omega$, and let $\mathscr{N}$ be the closed subset consisting of nilpotent structures. We shall see that 
$\mathscr{N}$ is the union of two components and that one of these is in fact a component of $\mathscr{C}$.

First we catalog the algebras in $\mathscr{N}$. Let $k$ denote $\Omega$ or an algebraically closed subfield, and let $\nu \in k$. Let $N_{\nu}$ be the $k$-algebra with basis $x_{1}, x_{2}, x_{3}$ and multiplications $x_{1} x_{2}=x_{3}, x_{2} x_{1}=\nu x_{3}$, with $x_{h} x_{i}=0$ otherwise. One has $N_{\nu}$ isomorphic to $N_{\mu}$ if and only if $\nu=\mu^{-1}$; cf. [2, p. 91]. By a direct computation, one sees that all but four of the three-dimensional nilpotent algebras over $k$ are of the form $N_{\nu}$. The exceptions are (here $u_{1}, u_{2}, u_{3}$ is a $k$-basis and products not listed or implied are zero):

$$
\begin{array}{ll}
\square: & \text { satisfying } \square^{2}=(0) \text { ("square-zero") } \\
C: & u_{1}^{2}=u_{2}, u_{1}^{3}=u_{3} \text { ("cyclic") } \\
J_{0}: & u_{1}^{2}=u_{3} \\
J_{1}: & u_{1}^{2}=u_{1} u_{2}=-u_{2} u_{1}=u_{3} .
\end{array}
$$

By computing the automorphism groups of the various algebras, one sees $\operatorname{dim} G \cdot N_{\nu}=5(\nu \neq-1)$ while $\operatorname{dim} G \cdot N_{-1}=3 ;$ also $\operatorname{dim} G \cdot C=6$, $\operatorname{dim} G \cdot J_{0}=4, \operatorname{dim} G \cdot J_{1}=5$. Further, if $\nu \in k$ and $\tau$ is transcendental over $k$, one may deform $N_{\nu}$ over $k$ into $N_{\tau}$.

Let $\mathscr{N}_{*}$ be the union of all orbits of the form $G \cdot N_{\nu}$ for $\nu \in \Omega$ and let $\mathscr{N}_{\#}$ denote its closure. We shall proceed as follows:

Lemma $6 . \mathscr{N}_{\#}$ is a subvariety of $\mathscr{N}$.

Lemma 7. $T_{\#}$ is the union of the orbits $G \cdot N_{\nu}$ with $\nu \in \Omega$ together with the three orbits $G \cdot \square, G \cdot J_{0}$ and $G \cdot J_{1}$.

Lemma 8. $\mathscr{N}$ is a reducible algebraic set with two components $\mathscr{N}_{\#}$ and $\mathscr{N} \cap \mathscr{C}^{\circ}$, where $\mathscr{C}_{\text {cm }}$ denotes the component of commutative structures in $\mathscr{C}$. The intersection of $\mathscr{N}$ and $\mathscr{N} \cap \mathscr{C}_{\mathrm{cm}}$ is the union of the orbits $G \cdot \square, G \cdot N_{1}, G \cdot J_{0}$ (and, in characteristic two, $G \cdot J_{1}$ ).

By combining the results of $\S 1$ with some "nondeformability" computations, we shall obtain finally:

Theorem 9. $\mathscr{N}_{\#}$ is a component of the algebraic set $\mathscr{C}=\mathscr{C}(3, \Omega)$; moreover $\mathscr{N}_{\sharp}$ carries no open orbit.

We prove these results in turn.

Proof of Lemma 6. It suffices to prove $\mathscr{N}_{*}$ irreducible. But this follows from the fact that $\mathscr{N}_{*}$ contains a subvariety isomorphic with $\Omega$ which intersects every orbit $G \cdot N_{\nu}$, namely the subvariety of 
points $c=\left(c_{h i j}\right)$ with entries $c_{123}=1, c_{213}=\nu$ and $c_{h i j}=0$ otherwise.

To obtain Lemma 7 we will apply the following observation, whose proof is straightforward:

Lemma 7a. Let $A$ and $B$ be k-algebras and let $A$ deform into $B$ in the sense of [2] (that is, there is a generic element $A_{t}$ of $a$ one-parameter family of deformations of $A$ which is K-isomorphic with $B_{K}$, where $\left.K=k((t))\right)$. Then the orbit $G \cdot A$ is contained in the closure $(=k$-closure) of the orbit $G \cdot B$.

Proof of Lemma 7. (i) We show that $\square$ and $J_{0}$ with scalars in a field $k$ deform into some $N_{\nu}, \nu \in k$, and then appeal to Lemma 7a. First, it is well-known that $\square$ deforms into any $k$-algebra. Now let $J_{0}$ have $k$-basis $u_{1}, u_{2}, u_{3}$ and deform as follows: $u_{1} * u_{1}=u_{1} u_{1}=u_{3}$, $u_{2} * u_{2}=u_{2} u_{2}=0, u_{1} * u_{2}=t u_{3}, u_{2} * u_{1}=u_{2} u_{1}=0$, other products zero. This multiplication gives a $K$-algebra $\left(J_{0}\right)_{t}$. Then the $K$-linear map $t u_{1}-u_{2} \rightarrow x_{1}, u_{2} \rightarrow x_{2}, t^{2} u_{3} \rightarrow x_{3}$ is a $K$-algebra isomorphism $\left(J_{0}\right)_{t} \rightarrow\left(N_{0}\right)_{K}$. By Lemma $7 \mathrm{a}, G \cdot J_{0} \subset \mathscr{N}_{\ddagger}$.

(ii) To show $G \cdot J_{1} \subset \mathscr{N}$, we deform $J_{1}$ over $k$ into $N_{\nu}$ with $\nu$ transcendental over $k$ and then argue geometrically. If the characteristic is not two, deform as follows: $u_{1} * u_{1}=u_{1} u_{1}=u_{3}, u_{1} * u_{2}=$ $(1+t) u_{3}, u_{2} * u_{1}=(-1+t) u_{3}$, other products zero as before. Then the map $u_{1}-(2 t)^{-1} u_{2} \rightarrow x_{1}, u_{2} \rightarrow x_{2}, u_{3} \rightarrow x_{3}$ determines a $K$-algebra isomorphism $\left(J_{1}\right)_{t} \rightarrow N_{\nu}$ where $\nu \in K$ equals $(t-1) /(t+1)$. In characteristic two we define $u_{2} * u_{1}=(1+t) z$ and $u_{h} * u_{i}=u_{h} u_{i}$ otherwise. In this case the map $t^{-1} u_{1}+t^{-2} u_{2} \rightarrow x_{1}, u_{2} \rightarrow x_{2}, t^{-1} u_{2} \rightarrow x_{3}$ determines a $K$-algebra isomorphism with $N_{\nu}$ where $\nu=1+t$.

Now in any characteristic each $k$-embedding $k(t) \rightarrow \Omega$ determines a structure $\bar{c}$ related to the above multiplication and the basis $u_{1}, u_{2}, u_{3}$. In each case $\bar{c}$ is an element of some $G \cdot N_{\nu}$, The set of all such $\bar{c}$ determines a $k$-rational curve in $\mathscr{N}_{\#}$ which contains the structure $c$ determined by the original multiplication $(t=0)$ and the basis $u_{1}, u_{2}, u_{3}$. But since $\mathscr{A}$ is a transformation space for $G$, it contains the entire orbit $G \cdot J_{1}$.

(iii) Finally, one sees that $G \cdot C$ is not a subset of $\mathscr{A} \#$ as follows: since $\operatorname{dim} G \cdot C=6$, the orbit is open in $\mathscr{N}_{\sharp}$, and so the algebras $N_{\nu}$ would deform into $C$ over suitable fields $k$. But only commutative algebras deform into $C$, a contradiction. Lemma 7 is proved.

Proof of Lemma 8. From Lemma 7 we see that $\mathscr{N}$ is the union of the irreducible $\mathscr{N}_{\#}$ and $\mathscr{A} \cap \mathscr{C}_{\mathrm{cm}}$. But the latter is the closure of the orbit $G \cdot C$, since one can deform $\square, J_{v}, N_{1}$ (and, in characteristic two, $J_{1}$ ) into $C$ over $k$ and then apply Lemma 7a. Thus $\mathscr{N} \cap \mathscr{C}_{c m}$ is irreducible, giving the lemma. 
Proof of Theorem 9. First we must consider the nonnilpotent algebras on $\mathscr{C}$. One computes directly that over an algebraically closed $k$ there are sixteen nonisomorphic nonnilpotent three-dimensional algebras. Of these, seven are rigid, whence the set $\mathscr{C}$ has at least seven components. Three of these seven components have dimensions 3,3 and 5 and hence cannot contain the six-dimensional $\mathscr{N}_{*}$. The other four rigid $k$-algebras are these: $S=k \oplus k \oplus k$, semi-simple; $U=$ the $2 \times 2$ upper-triangular matrices; $B$, consisting of those $3 \times 3$ matrices generated by the standard matrices $e_{11}, e_{22}, e_{13}$; and the opposite $B^{\prime}$ of $B$, generated by $e_{11}, e_{22}, e_{31}$. One computes that $\operatorname{dim} G \cdot S=9$, $\operatorname{dim} G \cdot B=\operatorname{dim} G \cdot B^{\prime}=8$ and $\operatorname{dim} G \cdot U=7$; these, therefore, are the dimensions of their components as well.

To show that $\mathscr{N}_{\#}$ is not contained in any of these four components, it suffices by $\S 1$ to show that $\mathscr{N}_{\#}$ carries algebras which do not deform into the rigid algebra dominating that component. Thus $\mathscr{N}_{\#}$ is not a subset of $\mathscr{C}_{c m}=\mathscr{C}(S)$, the component carrying semisimple $S$, since only commutative algebras deform into $S$. On the other hand, it is known that the algebra $N_{0}$ can be deformed into $U$ (see [1] for the formula). Thus the variety $\mathscr{N}_{\sharp}$ intersects the component $\mathscr{C}(U)$ nontrivially (that is, away from their common vertex) and might perhaps be contained in $\mathscr{C}(U)$. This possibility, and the possibility that $\mathscr{N}_{\#}$ is contained in the components $\mathscr{C}(B)$ or $\mathscr{C}\left(B^{\prime}\right)$, is ruled out by the following "nondeformation" result.

Lemma 9a. Let $k$ be any field. If $\nu \neq 0,-1$, then $N$, does not deform into $U, B$ or $B^{\prime}$.

Proof. Assume first that $N_{\nu}$ deforms into $U$; deformation into $B$ will be considered in (iii) below. (i) By Theorem 1 of [1], we may suppose that the deformed algebra $\left(N_{\downarrow}\right)_{t}$ has a $K$-basis

$$
u_{i}=\alpha_{1 i} x_{1}+\alpha_{2 i} x_{2}+\alpha_{3 i} x_{3} \quad(i=1,2)
$$

and $u_{3}=x_{3}$ with $\alpha_{h i} \in K$ such that the map $u_{1} \rightarrow e_{11}, u_{2} \rightarrow e_{22}, u_{3} \rightarrow e_{12}$ gives a $K$-algebra isomorphism $\left(N_{\nu}\right)_{t} \rightarrow U_{K}$.

We shall construct an equivalent deformation in which $\alpha_{21}=0$; this will simplify the computations of (ii) below. We may write $\alpha_{11}=a_{1} t^{q}(1+\cdots), \alpha_{21}=a_{2} t^{r}(1+\cdots)$ in $K=k((t))$. We first claim $q \neq r$. For assume that $q=r$, and note that $u_{1}^{\prime}=u_{1}-\alpha_{31} x_{3}$ is idempotent. Thus $t^{-q} u_{1}^{\prime} * t^{-q} u_{1}^{\prime}=t^{-2 q} u_{1}^{\prime}$. But a direct computation shows that this product is of the form $a_{1} a_{2}(1+\nu) x_{3}+$ (higher powers of $t$ with coefficients in $N_{\nu}$ ), a contradiction unless $\nu=-1$. Without loss then, $q<r$.

The promised equivalence can now be obtained as follows: let $\Phi_{t}$ 
denote the $K$-linear automorphism of the underlying $K$-space of $\left(N_{\downarrow}\right)_{t}$ given by $\Phi_{t}^{-1}\left(x_{1}\right)=x_{1}+\alpha_{21} \alpha_{11}^{-1} x_{2}, \Phi_{t}^{-1}\left(x_{2}\right)=x_{2}, \Phi_{t}^{-1}\left(x_{3}\right)=x_{3}$. Because $q<r$, $\Phi_{t}$ is of the form $\Phi_{t}(x)=x+$ (higher powers of $\left.t\right)$. Thus the multiplication $(x, y) \rightarrow \Phi_{t}\left(\Phi_{t}^{-1}(x) * \Phi_{t}^{-}(y)\right)$ gives a deformation of $N_{\nu}$ equivalent (see $[2$, p. 65]) to the $*$ multiplication. Moreover with this equivalent product the algebra $\left(N_{\nu}\right)_{t}$ admits the basis $u_{1}, u_{2}, u_{3}$ with $\alpha_{21}=0$ and multiplication table listed above.

(ii) In this equivalent product one now observes that $u_{1} * u_{3}=u_{3}$ implies $x_{1} * x_{3}=\alpha_{11}^{-1} x_{3}, u_{3} * u_{1}=0$ implies $x_{3} * x_{1}=0, u_{1}$ idempotent implies $x_{1} * x_{1}=\alpha_{11}^{-1} x_{1}$ and $u_{2} * u_{3}=0$ implies $x_{2} * x_{3}=-\alpha_{12}\left(\alpha_{11} \alpha_{22}\right)^{-1} x_{3}$.

Using these, one has $0=u_{2} * u_{1}=\alpha_{12} x_{1}+\alpha_{11} \alpha_{22}\left(\nu x_{3}+\right.$ (higher powers of $t$ with coefficients in $\left.N_{\nu}\right)$ ). This must be contradictory unless $\nu=0$. Thus $N_{\nu}$ cannot be deformed into $U$.

(iii) Now we show $N_{\nu}$ cannot be deformed into $B$ (nor, similarly, into $\left.B^{\prime}\right)$. Exactly as in (i) we would have a basis for $\left(N_{\nu}\right)_{t}$ of the form $\left\{u_{i}\right\}$ with $\alpha_{21}=0$ and a $K$-algebra isomorphism $\left(N_{\nu}\right)_{t} \rightarrow B_{K}$ given by $u_{1} \rightarrow e_{11}, u_{2} \rightarrow e_{22}, u_{3} \rightarrow e_{13}$. One now observes that all the conclusions of (ii) hold verbatim in the present case, since the product $u_{3} * u_{2}$ is not considered there. Thus the deformations are impossible as claimed, and Theorem 9 is established.

REMARK. The $N_{\nu}(\nu \neq 0,-1)$ are examples of semi-rigid algebras, that is, algebras whose radicals cannot decrease in size under deformation. See [1]. Deformation behavior on the component $\mathscr{N}_{\#}$ of $\mathscr{C}$ illustrates the fact that every finite-dimensional algebra deforms into a semi-rigid algebra, but not necessarily into one which is rigid.

\section{REFERENCES}

1. F. J. Flanigan, Straightening-out and semi-rigidity in associative algebras (submitted to Trans. Amer. Math. Soc.)

2. M. Gerstenhaber, On the deformation of rings and algebras, Ann. of Math. 79 (1964), 59-103.

3. A. Nijenhuis and R. Richardson, Cohomology and deformations in graded Lie algebras, Bull. Amer. Math. Soc. 72 (1966), 1-29.

4. B. L. von der Waerden, Modern Algebra, Vol. I, Frederick Ungar Co., New York, 1950.

5. R. J. Walker, Algebraic Curves, Princeton University Press, 1950.

6. A. Weil, Foundations of Algebraic Geometry, Amer. Math. Soc. Colloquium Publications, Vol. 29, Revised edition, 1962.

7. O. Zariski and P. Samuel, Commutative Algebra, Vol. 2, Van Nostrand Co., Princeton, 1960.

Received January 12, 1968. During the course of this research the author received partial support from NSF GP 6895. 



\section{PACIFIC JOURNAL OF MATHEMATICS}

EDITORS

H. ROYDEN

Stanford University

Stanford, California

R. R. Phelps

University of Washington

Seattle, Washington 98105
J. DugundJI

Department of Mathematics

University of Southern California

Los Angeles, California 90007

\section{RICHARD ARENS}

University of California

Los Angeles, California 90024

\section{ASSOCIATE EDITORS}
E. F. BeCKENBACH
B. H. NeUmanN
F. WOLF
K. YOSIDA

\section{SUPPORTING INSTITUTIONS}

UNIVERSITY OF BRITISH COLUMBIA CALIFORNIA INSTITUTE OF TECHNOLOGY UNIVERSITY OF CALIFORNIA MONTANA STATE UNIVERSITY UNIVERSITY OF NEVADA NEW MEXICO STATE UNIVERSITY OREGON STATE UNIVERSITY UNIVERSITY OF OREGON OSAKA UNIVERSITY UNIVERSITY OF SOUTHERN CALIFORNIA
STANFORD UNIVERSITY UNIVERSITY OF TOKYO UNIVERSITY OF UTAH WASHINGTON STATE UNIVERSITY UNIVERSITY OF WASHINGTON

AMERICAN MATHEMATICAL SOCIETY CHEVRON RESEARCH CORPORATION TRW SYSTEMS NAVAL WEAPONS CENTER 


\section{Pacific Journal of Mathematics \\ Vol. 27, No. $1 \quad$ January, 1968}

Willard Ellis Baxter, On rings with proper involution ............... 1

Donald John Charles Bures, Tensor products of $W^{*}$-algebras........... 13

James Calvert, Integral inequalities involving second order derivatives . . . . 39

Edward Dewey Davis, Further remarks on ideals of the principal class.... 49

Le Baron O. Ferguson, Uniform approximation by polynomials with integral

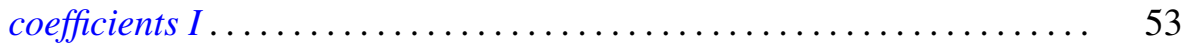

Francis James Flanigan, Algebraic geography: Varieties of structure

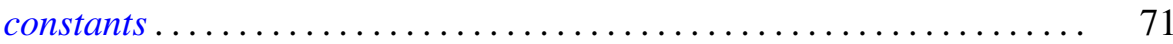

Denis Ragan Floyd, On QF -1 algebras ..................... 81

David Scott Geiger, Closed systems of functions and predicates ......... 95

Delma Joseph Hebert, Jr. and Howard E. Lacey, On supports of regular Borel measures ................................... 101

Martin Edward Price, On the variation of the Bernstein polynomials of a function of unbounded variation ........................ 119

Louise Arakelian Raphael, On a characterization of infinite complex matrices mapping the space of analytic sequences into itself........ 123

Louis Jackson Ratliff, Jr., A characterization of analytically unramified

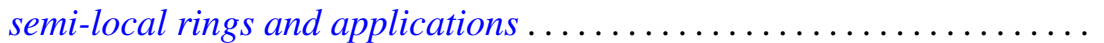

S. A. E. Sherif, A Tauberian relation between the Borel and the Lototsky transforms of series ................................ 145

Robert C. Sine, Geometric theory of a single Markov operator .......... 155

Armond E. Spencer, Maximal nonnormal chains in finite groups......... 167

Li Pi Su, Algebraic properties of certain rings of continuous functions .... 175

G. P. Szegô, A theorem of Rolle's type in $E^{n}$ for functions of the class $C^{1} \ldots 193$

Giovanni Viglino, A co-topological application to minimal spaces ........ 197

B. R. Wenner, Dimension on boundaries of $\varepsilon$-spheres ............... 201 